Drug-induced blood disorders

\title{
Effect of drugs on red cell membranes: Insights into normal red cell shape ${ }^{1}$
}

\author{
HARRY S. JACOB \\ From the Department of Medicine, University of Minnesota Medical School, Minneapolis
}

The distinguished speakers who have preceded me in this symposium have mainly ev'lored the role of drugs in ameliorating or causing disease. 1 should like to take a somewhat different tack. In the studies to be described drugs were used as probes of red cell physiology rather than as therapeutic agents. I shall concern myself with a class of drugs which react with the red cell membrane, and probably more specifically with a particular class of proteins in that membrane. By examining the effects of these drugs, my coworkers, Drs Yoshihito Yawata, Norburo Matsumoto, James White, and I have gained insights into the maintenance of the erythrocyte's normal biconcave shape, and moreover have gained an understanding of the abnormality of this shape in the common haemolytic disease, hereditary spherocytosis.

In our considerations of normal red cell shape we have become particularly interested in a class of proteins, loosely termed 'fibrous'. These proteins are characterized by their capacity to undergo reversible polymerization into structures with long axial ratios-structures which are depolymerized at low temperature or with exposure to drugs such as colchicine; in addition, fibrous proteins are denatured by the drugs vinblastine and strychnine. They may assume microtubular or microfilamentous conformations as exemplified by their appearances as mitotic spindles, cilia, and neurofilaments (Adelman, Borisy, Shelanski et al, 1968). In unicellular organisms, such as amoeba, they are frequently located at, or near, the cell membrane. Our interest in the possible association of such fibrous proteins with cell shape was provoked by observations in the platelet, in which microtubular structures, just beneath the cell membrane, are obvious when the platelet manifests its normal discoid shape (White, 1968). However, when platelets are cooled or exposed to any of the afore-

${ }^{1}$ Supported in part by grants from the National Institutes of Health, USPHS (AM-15730, HL-16833, and HL-15169), and from the Graduate School and Leukemia Research Fund of the University of Minnesota. mentioned drugs - vinblastine, colchicine, or strychnine-their microtubules disappear and concomitantly they become spheroidal (White, 1968). Analogous observations have been made in other cell types as diverse as amoeba and human fibroblasts in tissue culture.

It seemed reasonable to question whether similar membrane-associated fibrous proteins might be critical to red cell shape, since these cells also undergo reversible disc-sphere transformations (Nakao, Nakao, Yamazoe et al, 1961). Moreover, an equally plausible hypothesis would predict that a defect in this system might underlie hereditary spherocytosis, a disease in which red cells are abnormally shaped and rigid-abnormalities which cause them to undergo specific sequestration in the filter-meshwork of the spleen.

We were encouraged in this sequence of reasoning by the observations of Marchesi and Steers (1968), who demonstrated that the red cell membrane does indeed contain a protein which assumes a filamentous conformation. That is, about $20 \%$ of erythrocyte membrane protein, after solubilization at low ionic strength, aggregates into long microfilaments when ionic strength is increased. This protein, named 'spectrin', appears just at the interior of the intact red cell membrane as a filamentous network. The present studies, which have mainly utilized drugs which alter fibrous proteins and which have been partially reported elsewhere (Jacob, 1972; Jacob, Matsumoto, and Yawata, 1974), indicate that spectrin is crucial to red cell shape. Moreover, the integrity of this protein has been shown to require red cell metabolism, specifically ATP production and cyclic nucleotide availability. Finally, evidence will be presented that a defect in this system underlies the hereditary spherocytosis syndrome.

\section{Effects of Drugs on Shape of RBCs}

If normal, biconcave erythrocytes are exposed briefly to vinblastine, colchicine, or strychnine, their shape and deformability are strikingly altered (Jacob, Amsden, and White, 1972). Thus, under 


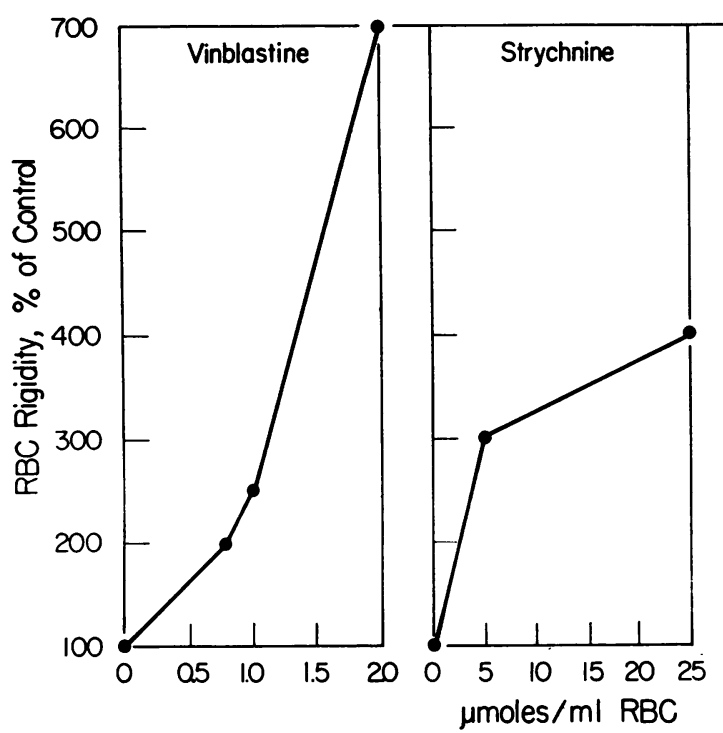

light microscopy normal cells become typical microspherocytes when briefly incubated with these compounds. Under scanning electron microscopy the three-dimensional appearance of these cells can be better appreciated, and when so examined, both cup-shaped (stomatocytes) and spheroidal cells are generated by the fibrous protein denaturants.

To survive normally in the circulation, erythrocytes must be highly deformable. Loss of deformability predicts their sequestration, especially in the constraining fenestrations of the splenic microvasculature. The fibrous protein-inhibiting drugs not only alter the shape of the erythrocyte but decrease their deformability as well (fig 1). Rigidity of red cells, measured by their resistance to filtration through artificial filters (Jacob, 1972), strikingly increases in the presence of vinblastine (left, fig 1), strychnine (middle, fig 1), and colchicine (right, fig 1).

As predicted, these abnormally rigid red cells have been shown to be vulnerable to entrapment and destruction in the spleen (Jacob et al, 1972), that is, when chromium-51 labelled, vinblastinetreated cells are reinjected into autologous donors, they are removed rapidly from the circulation and are predominantly accumulated by the spleen. The specificity of splenic sequestration is emphasized in that these same red cells survive normally when injected into a splenectomized subject.

\section{Cyclic Nucleotides and Shape of RBCs}

The abnormalities in morphology and deformability induced by these drugs, as well as other perturbations

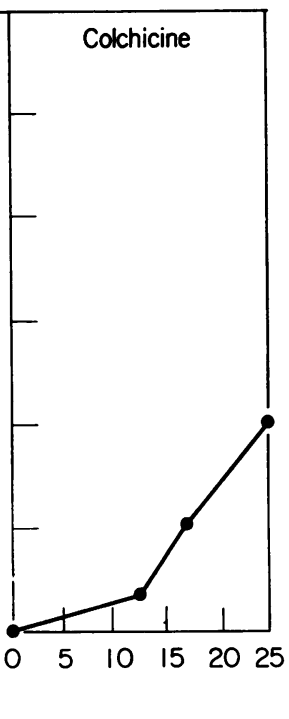

Fig 1 Effect of fibrous protein denaturants on $R B C$ rigidity. Red cells exposed for two hr at $37^{\circ} \mathrm{C}$ to vinblastine (left), strychnine (middle), or colchicine (right)drugs capable of interacting with fibrous proteins-become abnormally rigid (normal rigidity is set at 100 on the ordinate). Rigidity was determined by assay of the resistance of red cells to filtration through artificial filters.

in osmotic fragility, sodium permeability, and membrane fragmentability, which have also been described (Jacob, 1972; Jacob et al, 1972), are all characteristic of hereditary spherocytes. Such mimicry strengthened our interest in exploring the hypothesis that abnormalities in membrane fibrous proteins might underlie the hereditary spherocytosis syndrome.

In more critically examining the effect of these drugs on erythrocyte shape, we became aware that red cell metabolism was somehow crucial to membrane conformation and its alteration by the fibrous protein denaturants. For instance, cupping and sphering of erythrocytes was found not to occur in vinblastine-treated cells, unless red cell glycolysis, and probably ATP levels, were intact. Thus, if before the addition of vinblastine, red cells are treated for three hours with the glycolytic poison fluoride, so as to deplete them of most of their ATP content, they remain flat discs instead of becoming cup-shaped or spheroidal (left, fig 2); spiculated echinocytes, characteristic of metabolic depletion itself, are also seen. Following the removal of the fluoride, and reincubation of the cells in adenosine and glucose, so as to replenish ATP, the expected cup- and bowl-shaped cells form within 30 minutes and are well marked at 90 minutes (right, fig 2).

These studies suggest that membrane fibrous proteins are in some way modulated by the products of red cell metabolism, perhaps particularly by ATP and its byproducts. We became specially interested in the cyclic nucleotides, $3^{\prime} 5^{\prime}$ AMP (c-AMP) and $3^{\prime} 5^{\prime}$ GMP (c-GMP), since in tissue cultures these substances have been found to modulate the shape 


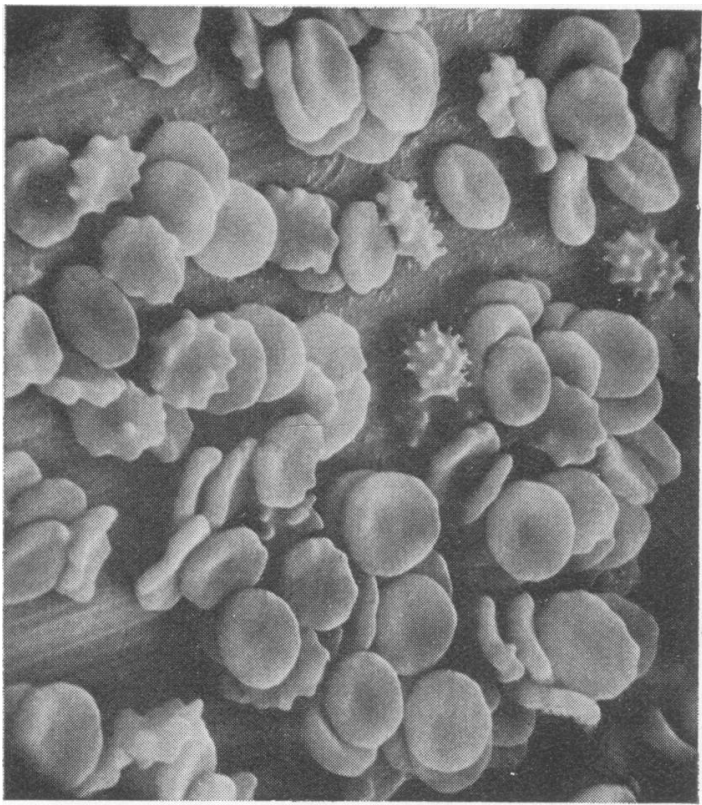

Left Red cells treated with fluoride for three $h r$ to deplete them of ATP and subsequently with vinblastine $(1 \mathrm{mM})$ for one hr do not become cup-shaped and spheroidal but remain flat biconcave discs and spiculated echinocytes.

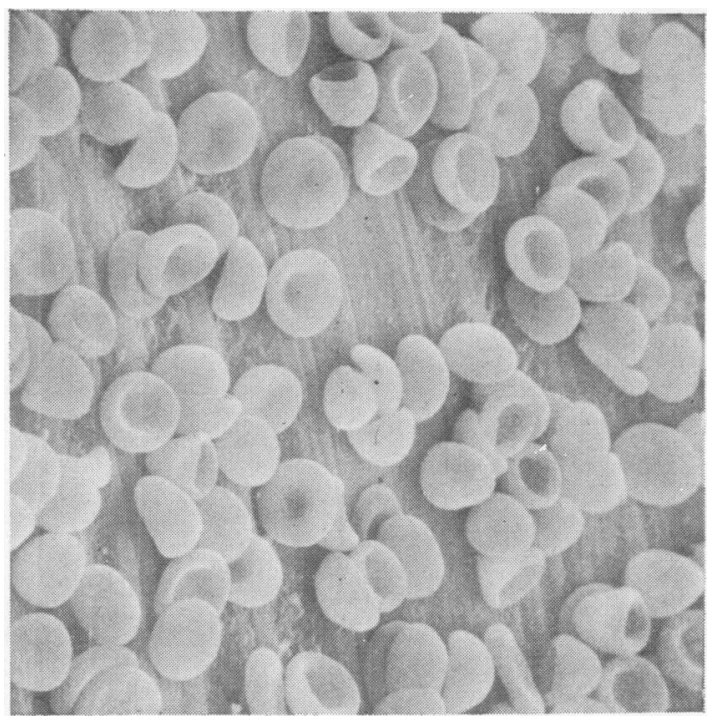

Right If fluoride is then washed away and the cells incubated in adenosine plus glucose to replenish ATP, progressive cupping and sphering ensure and are well-established after 90 minutes of such incubation.

Fig 2 Vinblastine-induced RBC cupping and sphering requires ATP. Scanning electron micrographs at magnification $\times$ 2000 are depicted.

and permeability of various cells and to do so by affecting fibrous proteins. For instance, vinblastine and colchicine have been shown to alter the shape and membrane transport of cultured, malignant, and axonal cells, but these alterations are specifically and competitively inhibited by the cyclic nucleotides (Hsie and Puck, 1971; Sheppard, 1971; Roisen, Murphy, and Braden, 1972; Kram and Tomkins, 1973; Johnson, Friedman, and Pastan, 1971). A role for cyclic nucleotides in red cell physiology has not been previously considered because their levels have been noted to be extremely low in these cells. Nevertheless, we were encouraged to seek a potential role for cyclic nucleotides in red cell conformation from a recent report that prostaglandin $E_{2}$, epinephrine, and isoproterenol alter erythrocyte deformability (Allen and Rasmussen, 1971). These agents, which are potent stimulants of cyclic nucleotide production in other cells, increase rigidity of human red cells when present in extremely low concentrations.

The following studies demonstrate that cyclic nucleotides do indeed modulate red cell membrane function, and do so by interaction with fibrous proteins. Thus, when observed under scanning electron microscopy c-GMP or c-AMP completely prevent the usual cupping and sphering of red cells induced by vinblastine or colchicine (left lower, fig 3). In contrast, the physiologically inactive cyclic 2-3 nucleotides, or non-cyclic $5^{\prime}$ GMP, do not prevent sphering and cupping (right lower, fig 3). This protective role of cyclic nucleotides on membrane integrity is further illustrated in figure 4 . The usual increase in osmotic fragility of red cells, which is induced by vinblastine, is virtually completely prevented by the addition of c-GMP; noncyclic $5^{\prime}$ GMP has little, if any protective effect (right, fig 4). Analogous results as regards sodium permeability are also obtainable (left, fig 4); that is, with utilization of double reciprocal plots, increasing concentrations of vinblastine can be shown to increase sodium leakiness of red cells progressively; c-GMP inhibits this induced leakiness, and does so with kinetics which indicate perfect competition between the cyclic nucleotide and vinblastine. Virtually identical results are obtained with c-AMP 

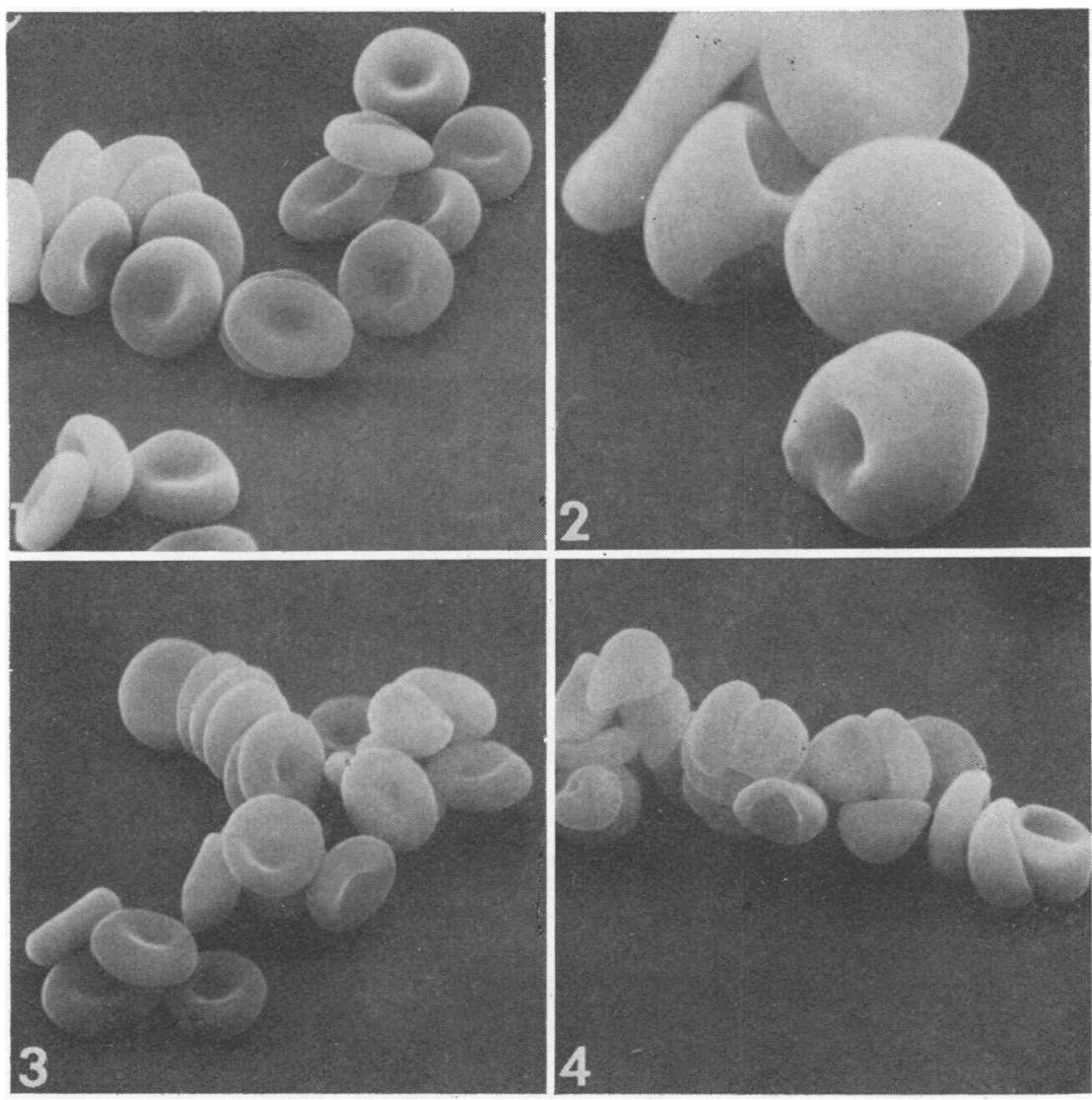

Fig 3 Inhibition of vinblastine-induced erythrocyte sphering and cupping by c-GMP: (1) untreated RBCs ( $\times 1900)$; (2) erythrocytic cups and spheres formed after two hours' incubation with vinblastine $(0.28 \mathrm{mM})$ at $37^{\circ} \mathrm{C}(\times 4200)$; (3) normal morphology persists when cyclic 3'5' GMP (10 mM) is added 15 minutes before vinblastine; (4) the addition of cyclic $2^{\prime} 3^{\prime}$ GMP (10 $m M)$ does not prevent cupping or sphering. Cyclic $3^{\prime} 5^{\prime}$ AMP is as effective as cyclic $3^{\prime} 5^{\prime}$ GMP in preventing changes in shape while non-cyclic 5' GMP is ineffective. as well, while the non-physiological 2-3 cyclic nucleotide congeners are inactive.

Finally, and perhaps most importantly, cyclic nucleotides also inhibit the splenic entrapment and destruction of vinblastine-treated red cells. As with human red cells, those from rats, if treated with vinblastine and reinfused after chromium-51 labelling, rapidly disappear from the circulation; concomitant with the loss of circulating radioactivity, labelled red cells accumulate solely in the rat spleens. However, if c-GMP is added to the red cells before vinblastine, subsequent red cell survival remains normal and splenic sequestration is prevented (Jacob et al, 1974).

\section{Role of Spectrin in the Shape of RBCs}

We next turned our attention to defining which protein mo'eties of the red cell membrane were involved in this interaction between cyclic nucleo- tides and fibrous proteins. Spectrin was found to be the main critical protein in this regard. Thus, if red cell membrane proteins are treated with vinblastine in concentrations required to produce red cell sphering, and the proteins then analysed by SDS gel electrophoresis, spectrin is the major component which disappears. The ability of cyclic nucleotides to inhibit vinblastine's deleterious effects on intact red cells is completely reflected at the subcellular level in that both c-GMP and c-AMP prevent this loss of spectrin from the gels, if added simultaneously with the vinblastine (Jacob et al, 1974).

In other tissues, cyclic nucleotides function by catalyzing a protein kinase reaction, which utilizes ATP to phosphorylate various protein substrates; others have demonstrated this reaction in intact red cells (Guthrow, Allen, and Rasmussen, 1972; Rubin, Erlichman, and Rosen, 1972). When this reaction was analysed further we found that spectrin is the major endogenous substrate for red cell 
Sodium Uptake

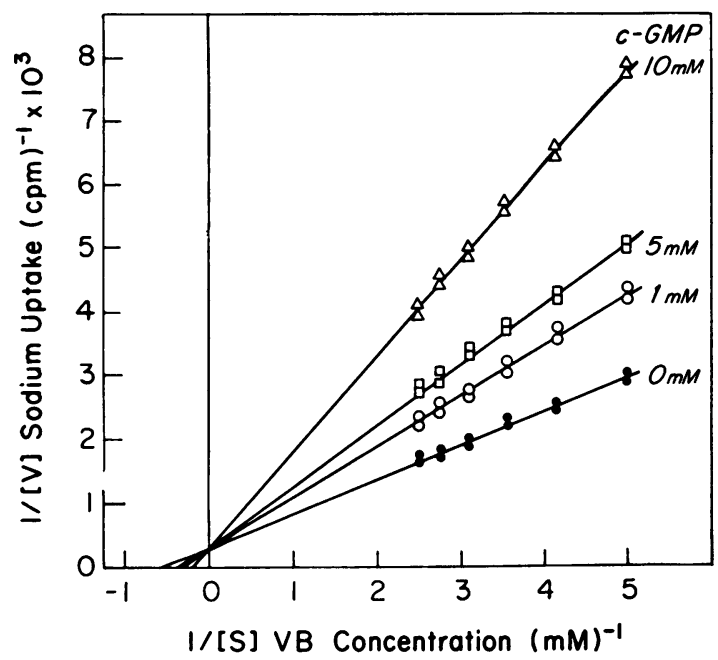

Osmotic Fragility

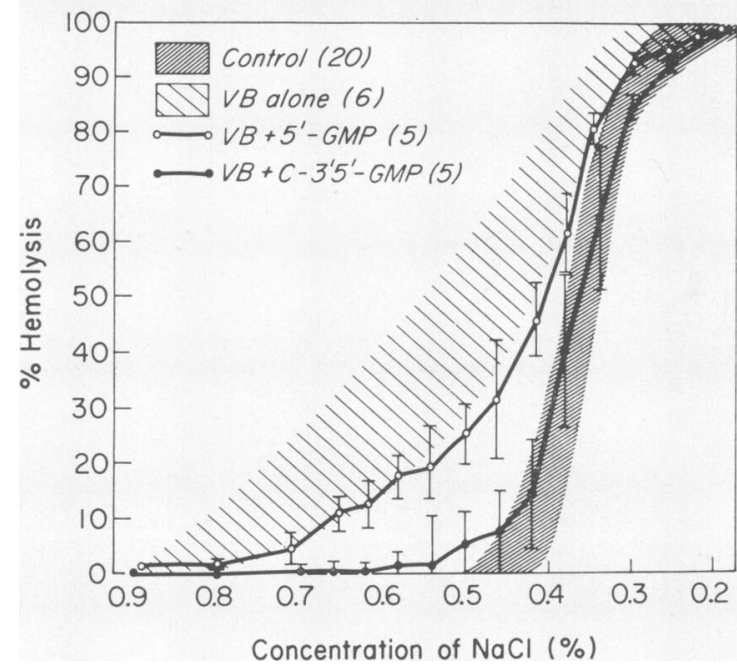

Fig 4 Cyclic $3{ }^{\prime} 5^{\prime}$ GMP competitively inhibits ${ }^{22} \mathrm{Na}$ uptake and prevents increased osmotic fragility of vinblastine $V B$ )-treated red cells. Left ${ }^{22} \mathrm{Na}$ uptake during incubation of cells for two hr at $37^{\circ} \mathrm{C}$ with various concentrations of vinblastine; cyclic 3'5' GMP inhibits the induced leakiness with competitive kinetics. Right Addition of $10 \mathrm{mM}$ cyclic $3^{\prime} 5^{\prime}$ GMP (closed circles) prevents increases in the osmotic fragility of red cells incubated with $0 \cdot 28 \mathrm{mM}$ vinblastine for two hours at $37^{\circ} \mathrm{C}$; non-cyclic $5^{\prime}$ GMP (open circles) has slight, if any, inhibitory effect. Brackets are $\pm S D$.

protein kinase, that is, when red cell membranes are phosphorylated by radioactive ATP in the presence of cyclic nucleotides, and the constituent proteins of the membranes analysed by SDS gels, the bulk of the radioactivity is associated with spectrin. Consistent with its ability to denature spectrin, vinblastine markedly inhibits this phosphorylation (Jacob et al, 1974). From these studies we may conclude that spectrin is a vinblastine-denaturable, fibrous protein which is phosphorylated by a cyclic nucleotidecatalyzed protein kinase.

To obtain further evidence that normal red cell shape and plasticity depend upon this phosphorylation of spectrin, we performed studies with heated red cells. It is well known that red cells briefly heated at $50^{\circ} \mathrm{C}$ become typical microspherocytes, similar to those seen in hereditary spherocytosis. Just as in the hereditary syndrome, heated cells are specifically sequestered in the spleen; in fact such cells have been utilized for clinical splenic scanning (Harris, McAlister, and Prankerd, 1957). When membrane proteins are extracted and electrophoresed from red cells briefly incubated at $50^{\circ} \mathrm{C}$, only spectrin is denatured. As would be predicted, a concomitant and progressive diminution in phosphorylation of membrane protein occurs with such heating. Moreover, phosphorylation both in the unstimulated state and with added cyclic nucleotides is diminished during heat-induced depletion of spectrin (Jacob et al, 1974).

Since ultracentrifugation studies by us (Jacob, Ruby, Overland, and Mazia, 1971), and electrophoretic analyses by others (Limber, Davis, and Bakerman, 1970) have suggested that mutant membrane proteins may be present in hereditary spherocytes, we were particularly interested in whether ATP-dependent membrane phosphorylation might be defective in this disease. Indeed we found such phosphorylation to be deficient as measured in membranes from six consecutive patients with hereditary spherocytosis. When compared with normal cells, a roughly $50 \%$ decrement in labelling was found, both with and without stimulation by cyclic nucleotides (Jacob et al, 1974). These results are similar to those recently reported by Greenquist and Shohet, who utilized slightly different techniques (Greenquist and Shohet, 1973). Since the most defective cells in this disease might be expected at any time to be entrapped in the spleen, it is of interest that the most severe abnormality of phosphorylation we have yet observed-that being only $30 \%$ of normaloccurred in hereditary spherocytes drained from the splenic pulp at the time of splenectomy of one of our patients. 
To summarize, spectrin, a filamentous protein located at the interior of red cell membranes, is specifically denatured by vinblastine or by brief heating at $50^{\circ} \mathrm{C}$. With loss of spectrin, sphering, rigidity, cation leakiness, and specific splenic sequestration of red cells result. By catalyzing spectrin's phosphorylation with ATP, cyclic AMP or cyclic GMP stabilizes the protein against denaturation. This phosphorylation reaction is inherently diminished in red cell membranes from patients with hereditary spherocytosis. From these studies we would conclude that spectrin underlies, and is critical to, normal red cell shape. For its proper function its phosphorylation by ATP is required in a reaction catalyzed by cyclic nucleotides. This process is genetically defective in hereditary spherocytosis.

\section{References}

Adelman, M.R., Borisy, G.G., and Shelanski, M.L., Weisenberg, R.C., and Taylor, E. W. (1968). Cytoplasmic filaments and tubules. Fed. Proc., 27, 1168.

cytosis red cells. (Abstr.), Blood, 42, 997.

Allen, J. E., and Rasmussen, H. R. (1971). Human red blood cellsprostaglandin $\mathrm{E}_{\text {, }}$ epinephrine, and isoproterenol alter deform: ability. Science, 174, 512-514.

Greenquist, A., and Shohet, S. B. (1973). ATP dependent phosphorylation of a membrane protein in normal and hereditary spherocytosis red cells. (Abstr) Blood, 42, 997.

Guthrow, C. E., Allen, J. E., and Rasmussen, H. (1972). Phosphorylation of an endogenous membrane protein by an endogenous, membrane-associated cyclic adenosine $3^{\prime}, 5^{\prime}$-monophosphatedependent protein kinase in human erythrocyte ghosts. J. biol. Chem., 247, 8145-8153.

Harris, I, M., McAlister, J. M., and Prankcrd, T. A. J. (1957). The relationship of abnormal red cells to the normal spleen. Clin. Sci., 16, 223-230.
Hsie, A. W., and Puck, T. T. (1971). Morphological transformation of Chinese hamster cells by dibutryl adenosine cyclic $3^{\prime}: 5^{\prime}$ monophosphate and testosterone. Proc. nat. Acad. Sci. (Wash), $68,358$.

Jacob, H. S. (1972). The abnormal red-cell membrane in hereditaryspherocytosis: evidence for the causal role of mutant micro filaments. Brit. J. Haemat., 23, Suppl., 35-44.

Jacob, H. S., Amsden, T., and White, J. (1972). Membrane microfilaments of erythrocytes: alteration in intact cells reproduces the hereditary spherocytosis syndrome. Proc. nat. Acad. Sci. (Wash.), 69, 471-474.

Jacob, H. S., Matsumoto, N., and Yawata, Y. (1974). Phosphorylation of a filamentous protein (spectrin) and RBC shape: defect in hereditary spherocytosis. Clin. Res., 22, 639a.

Jacob., H.S., Ruby, A., Overland, E.S., and Mazia, D. (1971). Abnormal membrane protein of red blood cells in hereditary spherocytosis. J. clin. Invest., 50, 1800-1805. of several morphological characteristic of normal fibroblasts in

Johnson, G. S., Friedman, R. M., and Pastan, I. (1971). Restoration of several morphological characters of normal fibroblasts in sarcoma cells treated with adenosine- $3^{\prime}: 5^{\prime}$-cyclic monophosphate and its derivatives. Proc. nat. Acad. Sci. (Wash.), 68, 425-429.

Kram, R., and Tomkins, G. M. (1973). Pleiotypic control by cyclic AMP: interaction with cyclic GMP and possible role of microtubules. Proc. nat. Acad. Sci. (Wash.), 70, 1659-1663.

Limber, G. K., Davis, R. F., and Bakerman, S. (1970). Acrylamide gel electrophoresis studies of human erythrocyte membrane. Blood, 36, 111-118.

Marchesi, V. T., and Steers, E., Jr. (1968). Selective solubilization of a protein component of the red cell membrane. Science, 159, 203-204.

Nakao, M., Nakao, T., Yamazoe, S., and Yoshikawa, H., (1961). Adenosine triphosphate and shape of erythrocytes. J. Biochem. 49, 487-492.

Roisen, F. J., Murphy, R. A., and Braden, W. G. (1972). Dibutyryl cyclic adenosine monophosphate stimulation of colcemidinhibited axonal elongation. Science, 177, 809-811.

Rubin, C. S., Erlichman, J., and Rosen, O. M. (1972). Cyclic adenosine $3^{\prime}, 5^{\prime}$ monophosphate-dependent protein kinase of human erythrocyte membranes. J. biol. Chem., 247, 6135-6139.

Sheppard, J. R. (1971). Restoration of contact-inhibited growth to transformed cells by dibutyryl adenosine $3^{\prime}: 5^{\prime}$-cyclic monophosphate. Proc. nat. Acad. Sci. (Wash.), 68, 1316-1320.

White, J. G. (1968). Fine structural alterations induced in platelets by adenosine diphosphate. Blood, 31, 604-622. 